
\title{
25 Research Square \\ The Diagnosis and Treatment of Low T3 Syndrome in Neurocritical Patients
}

\author{
Yihao Chen \\ Peking Union Medical College Hospital \\ Jianbo Chang \\ Peking Union Medical College Hospital \\ Rui Yin \\ Peking Union Medical College Hospital \\ Junxian Wen \\ Peking Union Medical College Hospital

\section{Baitao Ma} \\ Peking Union Medical College Hospital \\ Wei Zuo \\ Peking Union Medical College Hospital \\ Xiao Zhang \\ Peking Union Medical College Hospital \\ Junji Wei ( $\boldsymbol{\nabla}$ weijunji@pumch.cn ) \\ Peking Union Medical College Hospital
}

\section{Research article}

Keywords: low T3 syndrome, severe neurological disease, hormone replacement therapy, risk factors, mortality

Posted Date: December 19th, 2019

DOl: https://doi.org/10.21203/rs.2.19099/v1

License: (c) (i) This work is licensed under a Creative Commons Attribution 4.0 International License.

Read Full License 


\section{Abstract}

\section{Background}

Low serum T3 level is considered as a strong predictor of mortalities and poor prognosis in critical care patients. Few reports, however, focus on neurocritical patients. The application of hormone replacement therapy (HRT) in neurocritical patients with low T3 syndrome also remains controversial. We studied the role of low T3 state as a predictor in neurocritical patients and presented our experience of HRT from a single-center perspective.

\section{Methods}

From January 2012 to October 2018, a total of 32 neurocritical patients with low T3 syndrome were admitted to the neuro-intensive care unit (NICU) of Peking Union Medical College Hospital. Among them, 18 (56.25\%) patients received HRT (HRT group) since the diagnosis of low T3 syndrome, while the other 14 (43.75\%) patients did not (non-HRT group). We collected the clinical baseline and laboratory data of all the patients and conducted follow-up from 3 to 72 months. Overall survival was assessed by the Kaplan-Meier curve and compared by the log-rank test. Univariate and multivariate regression analysis was applied to estimate the prognostic power of HRT for mortality. We also performed the Mann-Whitney $U$ test or t-test to assess the influence of HRT on the final neurological function.

Results

The cohort consists of 32 patients, with an average Glasgow Coma Scale (GCS) of 6.41 (HRT=6.44 \pm 3.14 , non-HRT $=6.36 \pm 2.06)$. The neurocritical events include postoperative complications $(n=18)$, traumatic brain injury $(n=8)$, and spontaneous intracerebral hemorrhage $(n=6)$. A total of $15(46.87 \%)$ deaths were recorded $(H R T=7$, non-HRT $=8)$. In the HRT group, the low T3 situation in 5 patients $(33.3 \%)$ was corrected and $10(66.7 \%)$ were not. It turns out that the overall survival rate of the non-HRT group was significantly lower than that of the HRT group ( $P=0.034,16.445$ vs. 47.470 months). The non-HRT group has 3.322 times the mortality risk of the HRT group, according to univariate regression analysis, while the multivariate regression analysis showed no significant difference in mortality risk between the two groups ( $P=0.087, \mathrm{HR}=0.34095 \% \mathrm{Cl}$ 0.099-1.172). There was no significant difference in the short and long-term effects of HRT on neurological function (short-term GCS P $=0.587$, long-term GCS $P=0.419$, longterm GOS P=0.419).

\section{Conclusion}

Low T3 syndrome can significantly influence the prognosis of neurocritical patients. Therefore much attention should be paid to the changes in serum T3 level during treatment. Although it is unclear to what extent can HRT improve the short or long-term outcome of neurological function, it can significantly benefit the survival of neurocritical patients. 


\section{Introduction}

Low T3 syndrome has been described in the critical patients but without a prior history of thyroid disease. The most common manifestations include low triiodothyronine (T3) level, average or low thyroidstimulating hormone (TSH) level and increased reverse triiodothyronine (rT3) level.

Many studies have shown that a low T3 level was an independent predictor of poor prognosis in neurocritical patients ${ }^{1-3}$, and low T3 syndrome can influence various systems. For example, some studies found a rapid fall in serum T3 and T4 levels within 15 to 30 minutes after the initiation of cardiac bypass surgery and that change could last for days ${ }^{4}$. There is a strong positive correlation between low serum T3 and poor prognosis in patients with end-stage renal diseases ${ }^{5}$. Similarly, low T3 level is also a valid predictor of disease outcome in patients in intensive care units ${ }^{3,6}$. Patients with severe neurological diseases often have more complications and higher mortality rates, for which low T3 state is also an important prognostic indicator, as supported by plenty of evidence. Lieberman et al. found that the thyroid function of $87 \%$ of individuals with severe traumatic brain injury fell below the mid-normal value ${ }^{7}$. Many reports also showed that low T3 syndrome is one of the indicators of poor prognosis for cerebral infarction patients ${ }^{1,8}$. Their findings indicated the participation of central hypothyroidism in the most critical patients and it might be related to disturbance of thyroid hormone metabolism. Low T3 syndrome is common in patients with brain tumors and is positively correlated with shorter survival of glioma patients ${ }^{9}$. Despite the above, whether the thyroid hormone abnormalities are a physiological adaptation or a pathological change, is still debated ${ }^{10,11}$. Whether these changes are pathologic or physiologic and whether hormone replacement therapy (HRT) can benefit such patients, require further research. Here we aim to summarize the clinical features and outcomes of neurocritical patients with a low T3 level.

\section{Methods}

\subsection{Patient Population and Setting}

A retrospective review was performed on the medical records of patients admitted to the neurosurgery department of Peking Union Medical College Hospital between January 2012 and October 2018. We collected data from 1201 patients with triiodothyronine lower than the ordinary level and eliminated those with primary thyroid diseases. Totally 32 neurocritical patients with low T3 levels were included in the cohort, among which 18 received HRT and 14 did not. The HRT received contains a daily dose of $100 \mathrm{ug}$ of oral levothyroxine sodium tablet starting right after the diagnosis of low T3 states (treatment course: 18 days, median range: 5.75-30 days, $25-75$ th percentile). All patients were followed up through phone consultation for 3 months to 6 years. Table 1 summarizes the diagnosis and comorbidities of patients. The cohort was $50 \%$ male (median age $=46,38-54$ years, $25-75$ th percentile) and $50 \%$ female (median age $=56,46.7-74$ years, $25-75$ th percentile). In terms of neurocritical events, 18 cases of postoperative complications including intracranial hemorrhage $(n=3)$, subarachnoid hemorrhage $(n=2)$, cerebral infarction $(n=4)$, acute hydrocephalus $(n=3)$, central nervous system infection $(n=4)$ and severe cerebral 
edema $(n=5)$, were recorded, and eight cases of severe traumatic brain injury as well as six spontaneous intracerebral hemorrhage were diagnosed. During the progression of diseases, infection of the central nervous system $(n=14)$, pulmonary infection $(n=22)$ and heart failure $(n=8)$ also emerged. 
Clinical diagnoses of patients

Cerebral

Operation related complication*

Traumatic brain injury

Spontaneous intracerebral hemorrhage

CNS infection

Subarachnoid hemorrhage

Ischemic stroke

Hydrocephalus

Hyponatremia

Central diabetes insipidus

Central pontine myelinolysis

Respiratory

Pulmonary infection

22

Pulmonary embolism

Respiratory failure

Cardiovascular

Heart failure

Malignancy arrhythmia

High blood pressure

Intestinal

Gastrointestinal bleeding

Urinary tract

Urinary tract infection

Acute kidney injury

One patient can have more than one diagnosis, so the sum exceeds the absolute number of patients. *18 cases of postoperative complications include intracranial hemorrhage $(n=3)$, subarachnoid hemorrhage $(n=2)$, cerebral infarction $(n=4)$, acute hydrocephalus $(n=3)$, central nervous system infection $(n=4)$ and severe cerebral edema $(n=5)$. End-stage of death cases are often combined with multiple organ dysfunction.

Number of patients

18

8

6

14

3

1

4

1

4

1

1

4

8

5

11

1

4

5 
Endocrine

Type 2 diabetes

7

Blood system

Bacteremia

Chronic myeloid leukemia

1

One patient can have more than one diagnosis, so the sum exceeds the absolute number of patients. *18 cases of postoperative complications include intracranial hemorrhage $(n=3)$, subarachnoid hemorrhage $(n=2)$, cerebral infarction $(n=4)$, acute hydrocephalus $(n=3)$, central nervous system infection $(n=4)$ and severe cerebral edema $(n=5)$. End-stage of death cases are often combined with multiple organ dysfunction.

\subsection{Data Collection}

Data of all the patients with low T3 syndrome, which were adequately followed up, were analyzed. We recorded demographic information (name, gender, age, etc.), primary or secondary neurocritical events and their complications, and past disease history (e.g., cardiovascular diseases and infectious diseases). Total cortisol level test, liver function test, renal function test, complete blood cell analysis, coagulation function test and myocardial enzyme test were conducted using fasting blood samples to help evaluate the condition and progression of disease. We only took data from critical conditions into account if multiple test results exist. GCS at different stages were obtained from the medical records. Glasgow Outcome Scale (GOS) and the last GCS were obtained from follow-up.

\subsection{Laboratory Measurements}

All tests were conducted in the same laboratory using standard methods. The laboratory department of Peking Union Medical College Hospital has established its reference range. Serum fT3 (normal range: $1.80-4.10 \mathrm{pg} / \mathrm{ml}$ ), T3 (normal range: $0.66-1.92 \mathrm{ng} / \mathrm{ml}$ ), fT4 (normal range: $0.81-1.89 \mathrm{ng} / \mathrm{dl}$ ), T4 (normal range: $4.30-12.50 \mathrm{ug} / \mathrm{dl}$ ) and TSH (normal range: $0.38-4.34 \mu \mathrm{lU} / \mathrm{ml}$ ) levels were measured by enzymelinked immunosorbent assay (ELISA) from samples collected under critical conditions.

\subsection{Statistical Analysis}

Data analysis was performed using IBM SPSS 24.0 Statistical Software (SPSS Inc., Chicago, IL, United States). The Kolmogorov-Smirnov test was used to determine the distribution of continuous variables. For data of a non-normal distribution, results were presented as median and range and were compared using the Mann-Whitney $U$ test. For normally distributed data, results were reported as mean $\pm S D$ and were compared by t-test. Survival curves of HRT were calculated by the Kaplan-Meier method and differences in survival were estimated using the log-rank test. The differences were considered to be statistically significant when $\mathrm{P}<0.05$. We also performed a Cox proportional hazards model, determined relative risks for mortality using univariate and multivariate Cox regression analysis, and presented as 
hazard ratio (HR; 95\% Cl). Covariates tested in the Cox model were gender, age, BMI, fT3, GCS and HRT. Variables were included in the multivariate analysis if they had a P-value $<0.05$ in the univariate analysis or if the factors were regarded as clinically important confounders. When two-tailed $P<0.05$, the results were considered to be statistically significant.

\section{Results}

\subsection{Patients and Management}

A total of 16,830 patients attended the neurosurgery department of Peking Union Medical College Hospital and completed the thyroid function test during January 2012 and October 2019. Out of the 16,830 patients, 1201 (7.13\%) of them had lower-than-normal free triiodothyronine levels. Among them, we excluded $343(28.56 \%)$ outpatients and another $826(68.77 \%)$ patients were also excluded due to the absence of neurocritical events during hospitalization. Eventually, we admitted 32 (2.67\%) patients into the cohort of this study and none of them had primary thyroid diseases or were taking medications that could affect thyroid hormones. The median age of all the subjects was 53 years (range 41.25-64, 25-75th percentile) and the cohort was 50\% male and $50 \%$ female. All patients had a GCS no more than 11 during neurocritical events. The top 3 neurocritical events are postoperative complications ( 3 intracranial hemorrhage, 2 subarachnoid hemorrhages, 4 cerebral infarction, 3 acute hydrocephalus, 4 central nervous system infection and 5 severe cerebral edema), traumatic brain injury $(n=8)$, and spontaneous intracerebral hemorrhage $(n=6)$ (Fig. 1). Of the patients, $68.75 \%$ had pulmonary disease (22 cases of pulmonary infection, 1 pulmonary embolism and 4 respiratory failure) and $34.37 \%$ had cardiovascular disease ( 8 cases of heart failure, 5 malignant arrhythmias and 11 hypertension). We summarized the diagnosis information in Table 1.

\subsection{Lab Test Findings and Outcomes}

The baseline data and lab findings are as shown in Table 2. The HRT group had a median age of 46 years and that of the non-HRT group is 54 years. There was no significant statistical difference between the age of both groups $(P=0.059)$, nor between gender $(P=0.164)$ and between BMI $(P=0.319)$. All the subjects had average free triiodothyronine (fT3), free thyroxine (fT4), T3, T4, and TSH of $1.38 \mathrm{pg} / \mathrm{ml}, 0.94 \mathrm{ng} / \mathrm{ml}, 0.411 \mathrm{ng} / \mathrm{ml}, 4.58 \mu \mathrm{g} / \mathrm{dl}$, and $0.47 \mu \mathrm{lU} / \mathrm{ml}$, respectively. Twenty-two (52\%) patients had lower-than-normal fT4 levels, and 14 (33.3\%) patients had a normal TSH level. The non-HRT group had a higher median total cortisol concentration $(9.75 \mu \mathrm{g} / \mathrm{dl})$ than the HRT group $(4.66 \mu \mathrm{g} / \mathrm{dl})$, but with no statistical difference $(P=0.253)$. All 32 patients presented a median $G C S$ at 6 (HRT $=5.5$, nonHRT $=6)$ with no statistical difference between two groups either $(P=0.722)$. The mean GCS reached $8.50 \pm 0.73(H R T=8.17 \pm 1.07$, non-HRT $=8.93 \pm 0.98, P=0.587)$ when they were discharged. In the followup period, the mean GCS of all patients was $8.56 \pm 0.97$ (HRT $=9.33 \pm 1.28$, non-HRT $=7.57 \pm 1.49, \mathrm{P}=$ 0.419). 14 (43.8\%) patients encountered infection of central nervous system (HRT $=10$ or $55.55 \%$, nonHRT $=4$ or $28.57 \%)$. There was a total of $15(46.9 \%, \mathrm{HRT}=7$ or $38.88 \%$, non-HRT $=8$ or $57.14 \%)$ death in our study. 
Table 2

Baseline data

$\begin{array}{llll}\text { Characteristics } & \text { Total patients } & \text { HRT } & \text { Non-HRT } \\ & (n=32) & (n=18) & (n=14)\end{array}$

Demographic characteristics

\begin{tabular}{llll} 
Age-years* & $53(41.25-64)$ & $46(37.75-58.75)$ & $54(49-75.25)$ \\
\hline Male sex $(\%)$ & $50(n=16)$ & $38.8(n=7)$ & $64.28(n=9)$ \\
\hline BMI $(\mathrm{kg} / \mathrm{m} 2)$ & $24.20 \pm 0.59$ & $24.72 \pm 0.74$ & $23.53 \pm 0.76$
\end{tabular}

Thyroid hormones

\begin{tabular}{llll}
$\mathrm{fT} 3(\mathrm{pg} / \mathrm{ml})$ & $1.46 \pm 0.04$ & $1.44 \pm 0.06$ & $1.49 \pm 0.05$ \\
\hline $\mathrm{fT} 4(\mathrm{ng} / \mathrm{ml})$ & $1.04 \pm 0.16$ & $1.11 \pm 0.28$ & $0.95 \pm 0.09$ \\
\hline $\mathrm{T} 3(\mathrm{ng} / \mathrm{ml})$ & $0.40 \pm 0.02$ & $0.36 \pm 0.03$ & $0.45 \pm 0.04$ \\
$\mathrm{~T} 4(\mu \mathrm{g} / \mathrm{dl})$ & $4.72 \pm 0.43$ & $3.75 \pm 0.40$ & $5.90 \pm 0.71$ \\
$\mathrm{TSH}(\mu \mathrm{lU} / \mathrm{ml})$ & $1.12 \pm 0.37$ & $0.97 \pm 0.52$ & $1.33 \pm 0.53$
\end{tabular}

Laboratory findings

\begin{tabular}{llll}
\hline Blood cortisol(ug/dl)* & $4.83(1.54-15.86)$ & $4.66(1.32-12.04)$ & $9.75(2.10-22.24)$ \\
\hline Blood sugar $(\mathrm{mmol} / \mathrm{l})$ & $9.95 \pm 0.79$ & $10.18 \pm 1.11$ & $9.66 \pm 1.16$ \\
\hline Albumin $(\mathrm{g} / \mathrm{l})$ & $30.69 \pm 0.88$ & $31.39 \pm 1.37$ & $29.79 \pm 0.97$ \\
\hline Hemoglobin $(\mathrm{g} / \mathrm{l})$ & $90.75 \pm 3.27$ & $92.61 \pm 4.18$ & $88.36 \pm 5.32$ \\
\hline White blood cell $\left(\times 10^{9}\right)^{\star}$ & $15.86(12.93-20.54)$ & $17.66(13.09-21.32)$ & $13.71(10.10-17.36)$ \\
\hline Fibrinogen $(\mathrm{g} / \mathrm{l})$ & $3.98 \pm 0.36$ & $3.51 \pm 0.48$ & $4.59 \pm 0.53$
\end{tabular}

Clinical findings

\begin{tabular}{llll} 
GCS* & $6(5-8.75)$ & $5.50(3.75-10.25)$ & $6(5-8)$ \\
\hline GCS at discharge & $8.50 \pm 0.73$ & $8.17 \pm 1.07$ & $8.93 \pm 0.98$ \\
\hline GCS (follow-up) & $8.56 \pm 0.97$ & $9.33 \pm 1.28$ & $7.57 \pm 1.49$ \\
\hline GOS (follow-up) & $2.75 \pm 0.31$ & $3.00 \pm 0.41$ & $2.43 \pm 0.47$ \\
\hline CNS infection (\%) & $43.8(n=14)$ & $55.55(n=10)$ & $28.57(n=4)$ \\
\hline Survival outcome(death\%) & $46.9(n=15)$ & $38.88(n=7)$ & $57.14(n=8)$
\end{tabular}

*For data following non-normal distribution, results were expressed as median and range (median, 25-75th percentile). For data following a normal distribution, results were expressed as mean \pm SD. 


\subsection{Thyroid Function after Oral Administration of Levothyroxine Sodium}

Before receiving HRT (100 $\mu \mathrm{g}$ oral levothyroxine sodium daily), all patients were in accordance with typical low T3 syndrome manifestation, with serum fT3 levels lower than normal range. Fifteen patients in the HRT group were re-tested for thyroid function after treatment. The median time of the second test was 9 days (8-15 days, 25-75th percentile) after oral administration of levothyroxine sodium. Five $(33.3 \%)$ of them regained normal fT3 level (mean $=2.29 \pm 0.12)$ while the other $10(66.7 \%)$ still has an abnormally low level of fT3 (mean $=1.39 \pm 0.08)$. We compared the second results of fT3 $(1.69 \pm 0.51)$, fT4 $(1.00 \pm 0.29)$ and TSH $(1.14 \pm 2.96)$ from HRT group with baseline data $(1.41 \pm 0.06,1.15 \pm 0.34$, and $1.13 \pm 0.62$, respectively) using paired-samples t-test and found no statistical difference between them ( $P$ $=0.146, P=0.671, P=0.978$ ).

\subsection{Effects of Hormone Replacement Therapy 3.4.1 Survival Prognostic Relevance of Low T3}

Eighteen patients (HRT group) received oral administration of levothyroxine sodium (100 $\mu$ g per day, treatment course median is 18 days, range 5.75-30 days, 25-75th percentile). All 32 patients were followed up for 3 to 72 months. We compared the median overall survival of patients in the HRT group (n $=18$ ) with that of the non-HRT group $(n=14)$. Kaplan-Meier method and Cox regression survival analysis were used to calculate the mortality rate at 72 months of follow-up. The median survival of the HRT group was 47.470 months, which is significantly longer than that of the non-HRT group (median 16.445 months, $\mathrm{P}=0.034$ ). Figure 2 shows the Kaplan-Meier curves. In univariate regression analysis, HRT still made statistical differences, where the non-HRT group had 3.322 times the mortality risk of the HRT group $(P=0.043, H R=0.301,95 \% \mathrm{Cl}, 0.094-0.964)$, shown as Fig. 3 . Though the multivariate analysis, which includes age and GCS, indicated no statistical difference between the mortality risk of both groups, the hazard ratio $(0.340)$ between both groups can still be considered as a significant influencer of prognosis (Fig. 4).

\subsubsection{Neurological Prognostic Relevance of Low T3}

We compared the GCS at discharge and GCS and GOS at the last follow-up session between the HRT group $(n=18)$ and the non-HRT group $(n=14)$. The mean GCS score of the HRT patients at discharge was $8.17 \pm 1.07$ and the non-HRT ones recorded $8.93 \pm 0.98$. The t-test showed there was no significant statistical difference in short-term neurological outcomes between the two groups $(P=0.615)$. The mean GCS score at the last follow-up of the HRT patients was $9.33 \pm 1.28$ and the non-HRT was $7.57 \pm 1.49$. There was still no statistical difference between the two groups $(P=0.419)$. The mean GOS at the last follow-up of the two groups was $3.00 \pm 0.41$ and $2.43 \pm 0.47$, with no statistical difference, confirmed by ttest. (Fig. 5) 


\section{Discussion}

Reichlin and Protnay et al. had found that the thyroid hormone levels dropped in some critical patients without thyroid diseases back in the 1970s. Then, in 1982, Leonard Wartofsky and Bunnan from Washington Hospital proposed the concept of low T3 syndrome ${ }^{12}$. The low T3 syndrome is a disorder in thyroid hormone metabolism under various stress states, most commonly with the reduction in triiodothyronine as early as 24 hours after onset ${ }^{13}$. The primary mechanism behind the change is the inhibition of 5'-deiodinase. Usually, the free T4 level is among the normal range but could slightly exceed the limits. We observed a decline in T3 in our cohort at an average of 10 days after neurocritical events, and half of the subjects showed normal T4 levels. 5'-deiodinase activation induces the conversion of T4 into serum reverse triiodothyronine (rT3), which usually elevates in non-thyroid disease. However, many studies confirmed that the increase of rT3 could not accurately distinguish non-thyroid disease from hypothyroidism ${ }^{14}$.

The low T3 state was regarded as an independent predictor of mortalities in critical patients, especially for critical events and heart failure caused by any incidences ${ }^{15}$. Low T3 level, cardiac risk factors and mortality are strictly related ${ }^{16}$. The abnormal thyroid hormone levels were in companion with the failure of other systems or organs. According to a study on hormones in patients with end-stage renal disease undergoing hemodialysis, $44.3 \%$ of all 167 subjects had low T3 syndrome, which is also associated with mortalities of 6 months and 12 months $(P=0.007)^{5}$. The liver is involved in the conversion of tetraiodothyronine (T4) into triiodothyronine (T3), and patients with liver cirrhosis often had thyroid hormone abnormalities. A study demonstrated that nearly $67 \%$ of liver cirrhosis patients in intensive care units (ICU) had low T3 syndrome, and fT3 and fT4 levels may be used as predictors of mortality in such critical patients ${ }^{17}$. Wehmann et al. found that the incidence of low T3 syndrome in hematological malignancies was $54 \%{ }^{18}$. Wawrzyńskaet al. tested thyroid hormone concentration in severe respiratory failure patients in ICU and found that low T3 syndrome seems to be related to the decrease of PO2. Dying patients can have the lowest total T3 level, while the increase of TT3 serum concentration closely correlates with the improvement of the clinical state of patients ${ }^{12}$. Low fT3 levels have been interpreted as a physiological response aimed to reduce energy expenditure and minimize protein catabolism. Therefore, low T3 syndrome can be usually found in patients with malnutrition, fasting, and energy restrictions. A survival analysis of 669 hemodialysis patients with low T3 syndrome showed that nutritional status might serve as a "bridge" between low T3 levels and mortality. They also reported that age, cholesterol, and serum albumin concentration could be related to the extent of T3 level decline in different patients ${ }^{19}$. Therefore, low fT3 levels might also be an indicator of disease progression.

Neuroendocrine dysfunction (NED) is widespread in neurocritical patients. It has been reported that at least one NED was found in 35-50\% of individuals with severe traumatic brain injury $7,20,21$, and this may be related to the disorder of the hypothalamic-pituitary-target organ axis during acute progression. Stress is a defensive mechanism of the body to cope with the stressor to maintain the homeostasis. When under stress, the body's three major regulatory systems, i.e., the nervous system, the endocrine system and the 
immune system, are fully activated to protect the body by responding to internal and external stress. However, when the stress is prolonged or the homeostatic response is inadequate, this mechanism could lead to worse clinical conditions ${ }^{22}$. Thyroid hormones play an essential role in driving development and maintaining functions of the central nervous system $(C N S)^{23,24}$. The CNS could be impaired in thyroid disorders such as myxedema coma and thyrotoxic crisis. Therefore, alterations in thyroid hormone levels are often used as an explanation for some CNS dysfunctions ${ }^{25,26}$. Low T3 syndrome also affects the prognosis of neurological diseases such as acute stroke ${ }^{1}$, brain tumor ${ }^{9},{ }^{28}$. However, the CNS, as a complex functional network, interplays with multiple organs. Especially in neurocritical patients, multiorgan dysfunctions are prevalent. However, there are few clinical reports on patients with multi-system and multi-organ dysfunctions in companion with severe low T3 states.

As the acute progression comes to an end, the thyroid hormone levels may return to normal ${ }^{29}$. Maybe it implies that additional thyroid hormone supplements could improve the prognosis of low T3 patients. In our study, we evaluated the effects of the oral administration of levothyroxine sodium on survival outcomes and neurological outcomes in neurocritical patients with low-T3 syndrome. So far, several clinical studies on thyroid hormone replacement therapy for critical patients have been launched, focusing on topics including cardiac surgery ${ }^{30-32}$, malnutrition ${ }^{33,34}$, heart failure ${ }^{35,36}$, acute renal failure $^{37}$, premature infants with acute respiratory distress syndrome ${ }^{38}$. Unfortunately, most of them did not find any significant positive effects of HRT on prognosis, and no apparent harmful effects have been found either. Some small studies still demonstrate prospects for HRT, such as T3 supplementation in patients undergoing cardiac surgery, leading to less needs of inotropic support and better hemodynamic parameters ${ }^{39}$. There are scarcely any reports of thyroid hormone replacement therapy improving the prognosis of neurocritical patients with low T3 syndrome.

We divided 32 patients into 2 groups based on whether they received HRT (oral levothyroxine tablets, $100 \mu \mathrm{g})$. Then we performed Kaplan-Meier analysis and Cox regression analysis with 3 to 72 months follow-up and used mortality as indicators. From the results of Kaplan-Meier analysis, overall survival was significantly inferior in non-HRT patients than in HRT patients ( $P=0.034,16.445$ vs. 47.470 months). The univariate regression analysis showed that the mortality risk in the non-HRT group was 3.322 times higher than the HRT group $(P=0.043, H R=0.30195 \% \mathrm{Cl} 0.094-0.964)$. We incorporated the clinically significant variables (age and GCS) into multivariate analysis and the results showed no statistical difference in mortality risk between HRT and non-HRT group $(P=0.087, \mathrm{HR}=0.34095 \% \mathrm{Cl} 0.099-1.172)$. Although the P-value of the multivariate regression analysis was 0.087 , we still obtained a low-risk ratio (0.340, HRT vs. non-HRT group). Thus, we believe that oral hormone supplementation played a decisive role in improving prognosis and survival.

We sought to find evidence supporting that oral hormone supplementation could improve neurological outcomes in neurocritical patients with low serum T3. Descriptive analysis of short and long-term GCS or GOS showed that HRT patients have superior long-term GCS and GOS than non-HRT (GCS: $9.33 \pm 1.28$ vs. $7.57 \pm 1.49$; GOS: $3.00 \pm 0.41$ vs. $2.43 \pm 0.47$ ). However, the t-test showed no significant difference in short 
or long-term improvement in neurological functions between the two groups. Thyroid hormones enhanced the biological response to catecholamines ${ }^{40}$, which played a vital role in maintaining the stability of vascular volume and the function of endothelial cells. The velocity of cerebral arterial blood

flow is often positively correlated with thyroid hormone levels in vivo ${ }^{41}$. We believe that the normal thyroid hormone level is essential for maintaining craniocerebral hemodynamics stability. We observed an improvement of survival in patients who received hormone supplementation. Our study, however, did not obtain a positive result in neurological outcomes and that may result from the abysmal neurological prognosis in neurocritical patients. Besides, neurological assessments that are more precise than GCS or GOS could help achieve a more accurate result.

In our study, it seems that the oral hormone supplementation did not rapidly restore fT3 levels to a normal range. Among 15 patients who were re-tested for thyroid function in the HRT group, 5 (33.3\%) had been corrected, and the other $10(66.7 \%)$ had not. In spite of the result, we still believe that hormone supplementation makes its contribution to correcting the low T3 state. A prospective study treated patients with low T3 syndrome and ischemic or non-ischemic dilated cardiomyopathy with intravenous infusion of synthetic I-T3 for 3 days (initial dose: $20 \mu \mathrm{g} / \mathrm{m}^{2} / \mathrm{d}$ ) and found a rapid increase in free T3 level as well as a significant improvement in neuroendocrine profile and ventricular performance ${ }^{36}$. Therefore, proper hormone administration and dosage and a dynamic fT3 concentration monitoring were considered beneficial for the correction of low serum T3 in critical patients. Indeed, for critical patients, or more specifically, neurocritical patients, the exact relationship between the improvement of prognosis and complete correction of low T3 states requires more research and more support from evidence-based medicine.

\section{Study Limitations}

This study inevitably has some limitations. Firstly, as a retrospective research, selective bias is hard to avoid. Secondly, the actual subjects that met the criteria of both neurocritical and low T3 levels are insufficient in quantity, together with complicated individual conditions as well as limited variables, making it hardly possible to take all potential confounders into account properly. Thirdly, some patients in the HRT group did not receive continuous and dynamic monitoring of fT3 levels, leading to incomplete data. Also, finally, more refined and accurate means of evaluation are preferred to assess the impact of HRT on neurological outcomes better.

\section{Conclusion}

Our study demonstrated that hormone replacement therapy has a significant impact on prognosis and survival on neurocritical patients with low T3 syndrome but has no apparent influence on short or longterm neurological outcomes. We appeal more support from evidence-based medicine to help verify the effectiveness of HRT as a new approach to better treat neurocritical patients. 


\section{List Of Abbreviations}

\begin{tabular}{|l|l|}
\hline ALT & Alanine aminotransferase \\
\hline APTT & Activated partial thromboplastin time \\
\hline AST & Aspartate amino transferase \\
\hline BMI & Body Mass Index \\
\hline fT3 & Free triiodothyronine \\
\hline fT4 & Free thyroxine \\
\hline GCS & Glasgow coma score \\
\hline GOS & Glasgow prognostic score \\
\hline HRT & Hormone replacement therapy \\
\hline NICU & Neuro-intensive care unit \\
\hline PT & Prothrombin time \\
\hline TSH & Thyroid-stimulating hormone \\
\hline
\end{tabular}

\section{Declarations}

\section{Acknowledgments}

We would like to extend special thanks to Dr. Junji Wei for performing the emergency

surgical procedures and guiding the perioperative treatment.

\section{Funding}

There are no funds.

Data Availability Statement:

All data generated or analyzed during this study are available from the corresponding author on reasonable request.

\section{Authors' contributions}

Yihao Chen and Junji Wei designed and analyzed the data. All authors read and approved the final text.

\section{Ethics Approval and Patient Consent}

This study was approved by the Ethics Committee of Peking Union Medical College Hospital (PUMCH) and a waiver of informed consent was granted.

\section{Consent for publication}

Not applicable.

\section{Competing interests}

The authors declare no competing interests. 


\section{Author details}

${ }^{1}$ Dept. of Neurosurgery, Peking Union Medical College Hospital『Chinese Academy of Medical Sciences \& Peking Union Medical College; NO.1 Shuaifuyuan Hutong of Dongcheng District, Beijing, 100730 P. R. China;

${ }^{2}$ Dept. of Vascular surgery, Peking Union Medical College Hospital『Chinese Academy of Medical Sciences \& Peking Union Medical College; NO.1 Shuaifuyuan Hutong of Dongcheng District, Beijing, 100730 P. R. China

${ }^{3}$ Dept. of Pharmacy, Peking Union Medical College Hospital『Chinese Academy of Medical Sciences \& Peking Union Medical College; NO.1 Shuaifuyuan Hutong of Dongcheng District, Beijing, 100730 P. R. China

\section{References}

1. Alevizaki M, Synetou M, Xynos K, Pappa T, Vemmos KN. Low triiodothyronine: a strong predictor of outcome in acute stroke patients. Eur J Clin Invest. 2007;37(8):651-657.

2. lervasi G, Pingitore A, Landi P, et al. Low-T3 syndrome: a strong prognostic predictor of death in patients with heart disease. Circulation. 2003;107(5):708-713.

3. Wartofsky L, Burman KD. Alterations in thyroid function in patients with systemic illness: the "euthyroid sick syndrome". Endocr Rev. 1982;3(2):164-217.

4. Farwell AP. Nonthyroidal illness syndrome. Curr Opin Endocrinol Diabetes Obes. 2013;20(5):478-484.

5. Horacek J, Dusilova SS, Kubisova M, et al. Thyroid hormone abnormalities in hemodialyzed patients: low triiodothyronine as well as high reverse triiodothyronine are associated with increased mortality. Physiol Res. 2012;61(5):495-501.

6. Peeters RP, Wouters PJ, van Toor H, Kaptein E, Visser TJ, Van den Berghe G. Serum 3,3',5'triiodothyronine (rT3) and 3,5,3'-triiodothyronine/rT3 are prognostic markers in critically ill patients and are associated with postmortem tissue deiodinase activities. J Clin Endocrinol Metab. 2005;90(8):4559-4565.

7. Lieberman SA, Oberoi AL, Gilkison CR, Masel BE, Urban RJ. Prevalence of neuroendocrine dysfunction in patients recovering from traumatic brain injury. $J$ Clin Endocrinol Metab. 2001;86(6):2752-2756.

8. Ambrosius W, Kazmierski R, Gupta V, et al. Low free triiodothyronine levels are related to poor prognosis in acute ischemic stroke. Exp Clin Endocrinol Diabetes. 2011;119(3):139-143.

9. Bunevicius A, Deltuva VP, Tamasauskas S, et al. Preoperative low tri-iodothyronine concentration is associated with worse health status and shorter five year survival of primary brain tumor patients. Oncotarget. 2017;8(5):8648-8656. 
10. Farwell AP. Thyroid hormone therapy is not indicated in the majority of patients with the sick euthyroid syndrome. Endocr Pract. 2008;14(9):1180-1187.

11. Pappa TA, Vagenakis AG, Alevizaki M. The nonthyroidal illness syndrome in the non-critically ill patient. Eur J Clin Invest. 2011;41(2):212-220.

12. Wawrzynska L, Sakowicz A, Filipecki S. [Euthyroid sick syndrome in patients with respiratory failure]. Pneumonol Alergol Pol. 1996;64 Suppl 2:193-199.

13. Lee S, Farwell AP. Euthyroid Sick Syndrome. Compr Physiol. 2016;6(2):1071-1080.

14. Burmeister LA. Reverse T3 does not reliably differentiate hypothyroid sick syndrome from euthyroid sick syndrome. Thyroid. 1995;5(6):435-441.

15. Wang F, Pan W, Wang H, Wang S, Pan S, Ge J. Relationship between thyroid function and ICU mortality: a prospective observation study. Crit Care. 2012;16(1):R11.

16. Koo HM, Kim CH, Doh FM, et al. The impact of low triiodothyronine levels on mortality is mediated by malnutrition and cardiac dysfunction in incident hemodialysis patients. Eur J Endocrinol. 2013;169(4):409-419.

17. Teiger $E$, Menasche $P$, Mansier P, et al. Triiodothyronine therapy in open-heart surgery: from hope to disappointment. Eur Heart J. 1993;14(5):629-633.

18. Wehmann RE, Gregerman RI, Burns WH, Saral R, Santos GW. Suppression of thyrotropin in the lowthyroxine state of severe nonthyroidal illness. N Engl J Med. 1985;312(9):546-552.

19. Ozen KP, Asci G, Gungor O, et al. Nutritional state alters the association between free triiodothyronine levels and mortality in hemodialysis patients. Am J Nephrol. 2011;33(4):305-312.

20. Aimaretti G, Ambrosio MR, Di Somma C, et al. Traumatic brain injury and subarachnoid haemorrhage are conditions at high risk for hypopituitarism: screening study at 3 months after the brain injury. Clin Endocrinol (Oxf). 2004;61(3):320-326.

21. Kelly DF, Gonzalo IT, Cohan P, Berman N, Swerdloff R, Wang C. Hypopituitarism following traumatic brain injury and aneurysmal subarachnoid hemorrhage: a preliminary report. $J$ Neurosurg. 2000;93(5):743-752.

22. von Haehling S, Doehner W, Anker SD. Nutrition, metabolism, and the complex pathophysiology of cachexia in chronic heart failure. Cardiovasc Res. 2007;73(2):298-309.

23. Dussault JH, Ruel J. Thyroid hormones and brain development. Vitamins \& Hormones. 2005;71(3):95-122.

24. Kassem NA, Deane R, Segal MB, Preston JE. Role of transthyretin in thyroxine transfer from cerebrospinal fluid to brain and choroid plexus. American Journal of Physiology Regulatory Integrative \& Comparative Physiology. 2006;291(5):R1310-R1315.

25. Olsson T, Asplund K, Hägg E. Pituitary-thyroid axis, prolactin and growth hormone in patients with acute stroke. J Intern Med. 2010;228(3):287-290.

26. Solter M, Katalinic D, Vargek-Solter V, et al. Brain tumor as a prototype of severe brain lesion in patients with "low T3 syndrome". Acta Clin Croat. 2012;51(2):215-219. 
27. Sheu JJ, Kang JH, Lin HC, Lin HC. Hyperthyroidism and risk of ischemic stroke in young adults: a 5year follow-up study. Stroke. 2010;41(5):961-966.

28. Bunevicius A, Deltuva V, Tamasauskas S, Tamasauskas A, Laws EJ, Bunevicius R. Low triiodothyronine syndrome as a predictor of poor outcomes in patients undergoing brain tumor surgery: a pilot study: clinical article. J Neurosurg. 2013;118(6):1279-1287.

29. Economidou F, Douka E, Tzanela M, Nanas S, Kotanidou A. Thyroid function during critical illness. Hormones (Athens). 2011;10(2):117-124.

30. Bennett-Guerrero E, Jimenez JL, White WD, D'Amico EB, Baldwin BI, Schwinn DA. Cardiovascular effects of intravenous triiodothyronine in patients undergoing coronary artery bypass graft surgery. $\mathrm{A}$ randomized, double-blind, placebo- controlled trial. Duke T3 study group. JAMA. 1996;275(9):687692.

31. Klemperer JD, Klein I, Gomez M, et al. Thyroid hormone treatment after coronary-artery bypass surgery. N Engl J Med. 1995;333(23):1522-1527.

32. Vermes I, Beishuizen A. The hypothalamic-pituitary-adrenal response to critical illness. Best Pract Res Clin Endocrinol Metab. 2001;15(4):495-511.

33. Gardner DF, Kaplan MM, Stanley CA, Utiger RD. Effect of tri-iodothyronine replacement on the metabolic and pituitary responses to starvation. N Engl J Med. 1979;300(11):579-584.

34. Koppeschaar HP, Meinders AE, Schwarz F. Metabolic responses in grossly obese subjects treated with a very-low-calorie diet with and without triiodothyronine treatment. Int J Obes. 1983;7(2):133141.

35. Moruzzi P, Doria E, Agostoni PG. Medium-term effectiveness of L-thyroxine treatment in idiopathic dilated cardiomyopathy. Am J Med. 1996;101(5):461-467.

36. Pingitore A, Galli E, Barison A, et al. Acute effects of triiodothyronine (T3) replacement therapy in patients with chronic heart failure and low-T3 syndrome: a randomized, placebo-controlled study. $J$ Clin Endocrinol Metab. 2008;93(4):1351-1358.

37. Acker CG, Singh AR, Flick RP, Bernardini J, Greenberg A, Johnson JP. A trial of thyroxine in acute renal failure. Kidney Int. 2000;57(1):293-298.

38. Osborn DA. Thyroid hormones for preventing neurodevelopmental impairment in preterm infants. Cochrane Database Syst Rev. 2001(4):D1070.

39. Novitzky D, Cooper DK. Thyroid hormone and the stunned myocardium. J Endocrinol. 2014;223(1):R1-R8.

40. Franchini M. Hemostasis and thyroid diseases revisited. J Endocrinol Invest. 2004;27(9):886-892.

41. Sobky MAE, Shazly E, Darwish AK, Davies T, Griffin K, Keshaven MS. Anterior pituitary response to thyrotrophin releasing hormone in senile dementia (Alzheimer type) and elderly normals. Acta Psychiat Scand. 2010;74(1):13-17. 


\section{Figures}

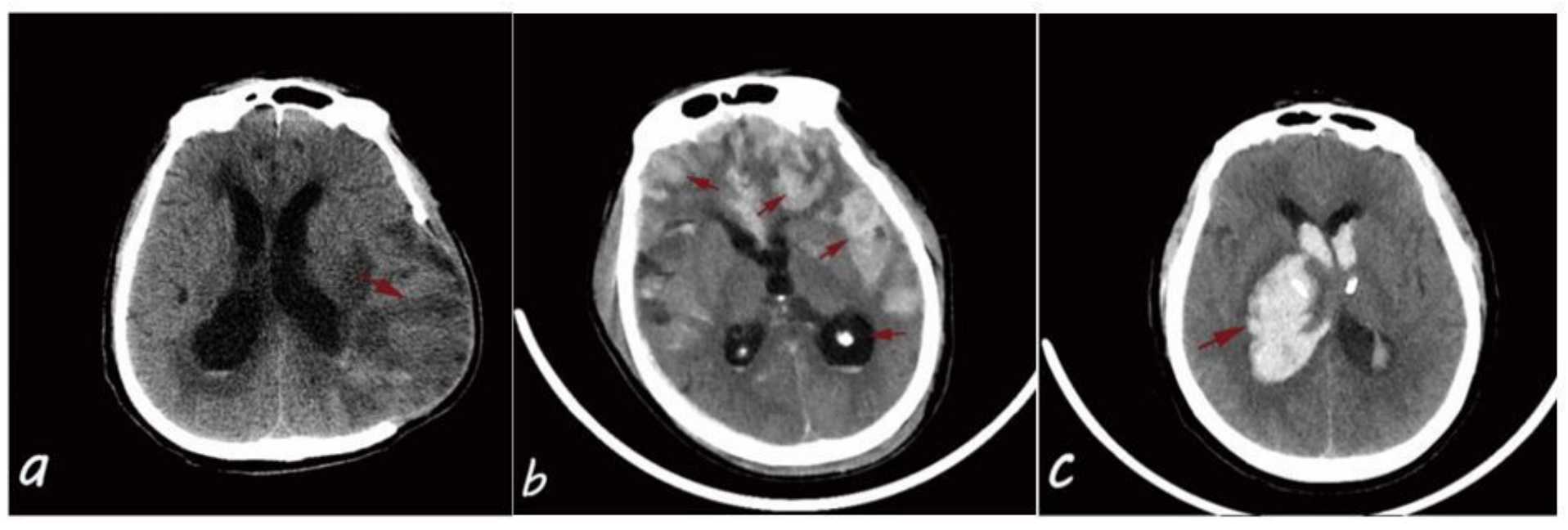

Figure 2

Imaging data of 3 death patients under different neurocritical events. a. Postoperative changes in glioblastoma, brain edema in the operation area, part of the tissue bulged outward. (Postoperative complication) b. High-density shadows were seen in the bilateral frontal, temporal, parietal lobe and ventricles. The ambient cistern was unclear. (Severe traumatic brain injury) c. A mass-like high-density shadow was seen in the right basal ganglia and combined with intraventricular hemorrhage. (Spontaneous intracerebral hemorrhage) 


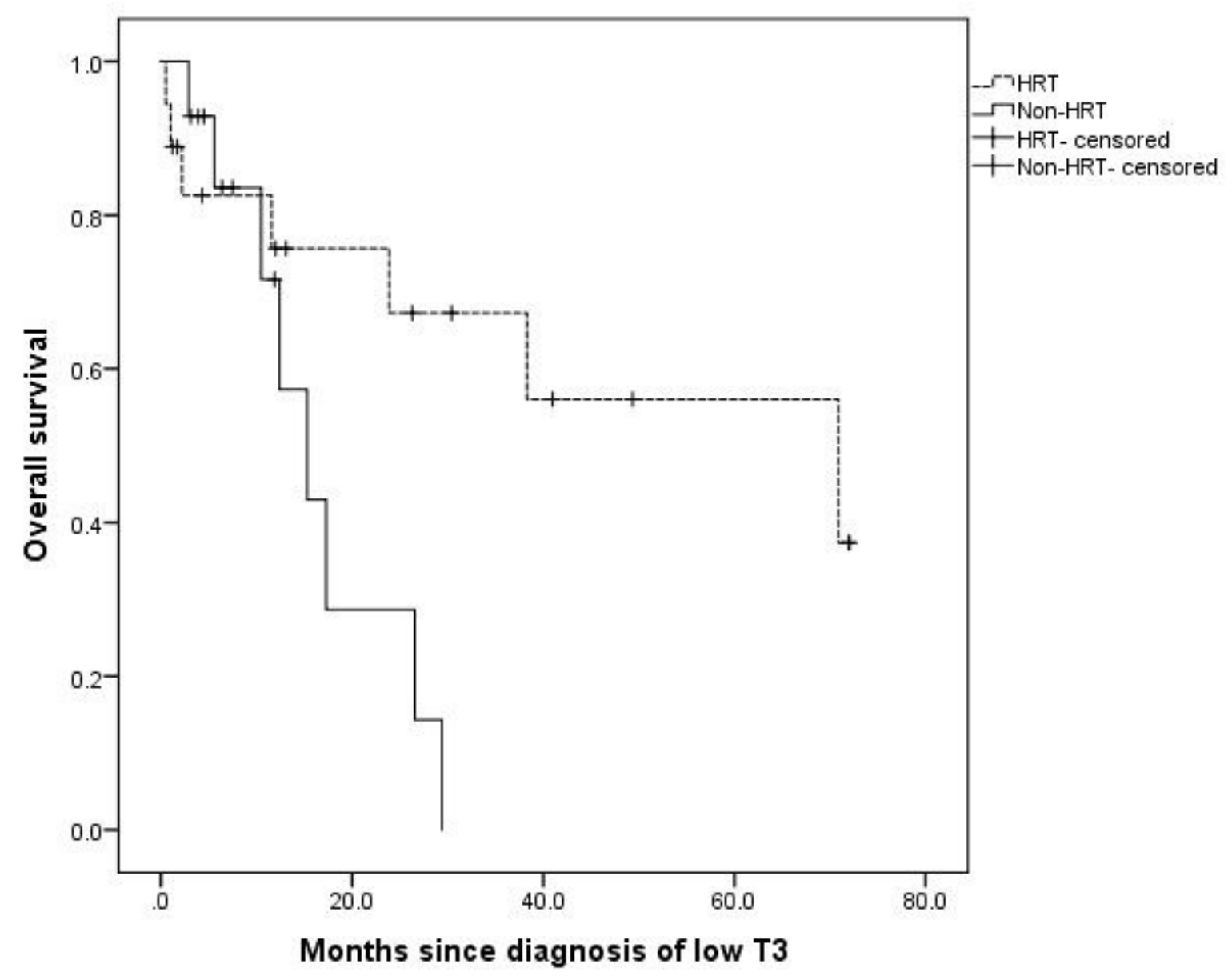

Figure 4

Kaplan-Meier plot of overall survival measured from the time of initial diagnosis of low T3 in critical patients. Median overall survival in non-HRT patients was significantly shorter than in HRT patients: 16.445 vs. 47.470 months, $P=0.034$. 


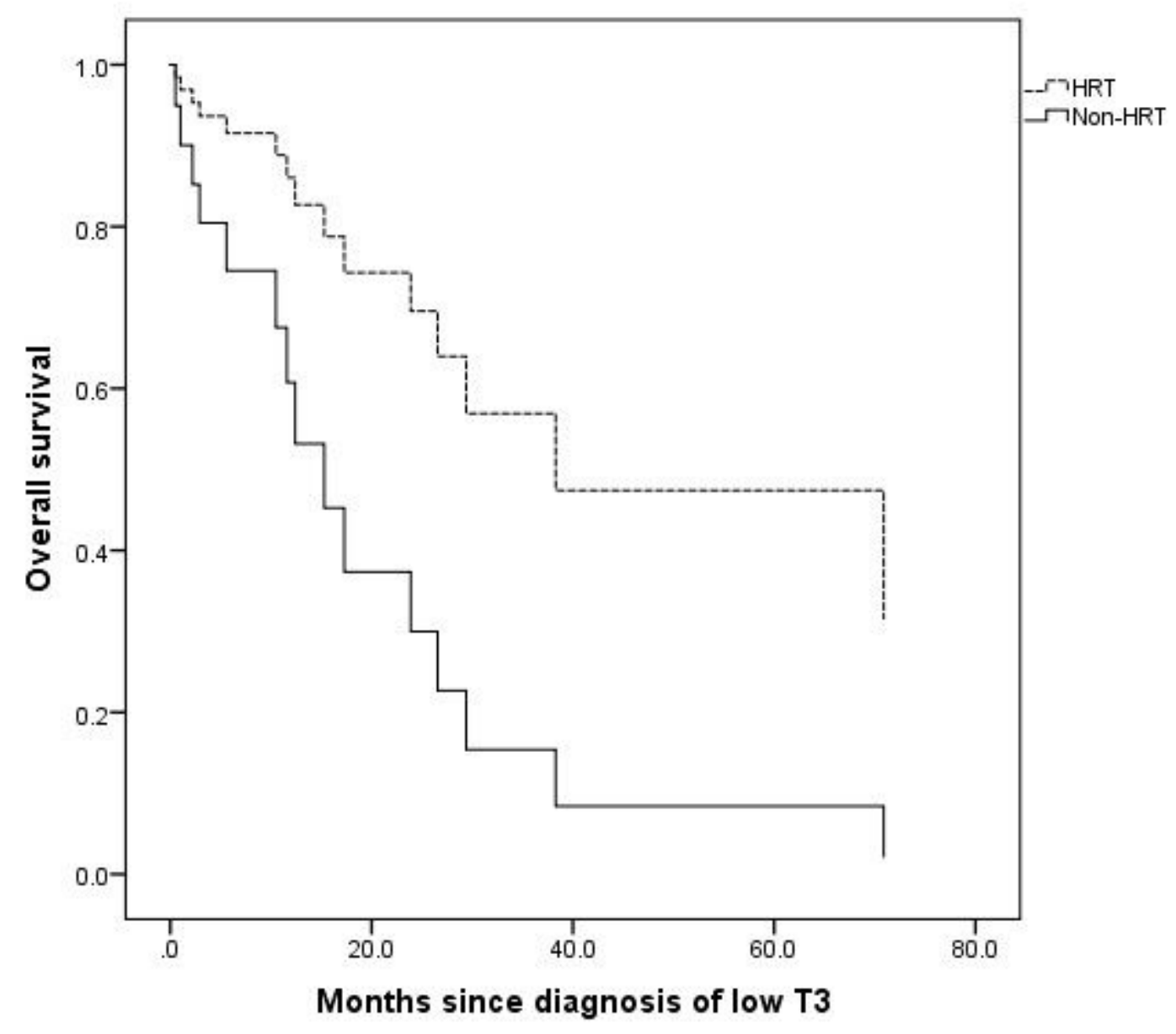

Figure 6

Univariate regression analysis showed the mortality risk in the non-HRT group was 3.322 times higher than HRT group, $\mathrm{P}=0.043$. 


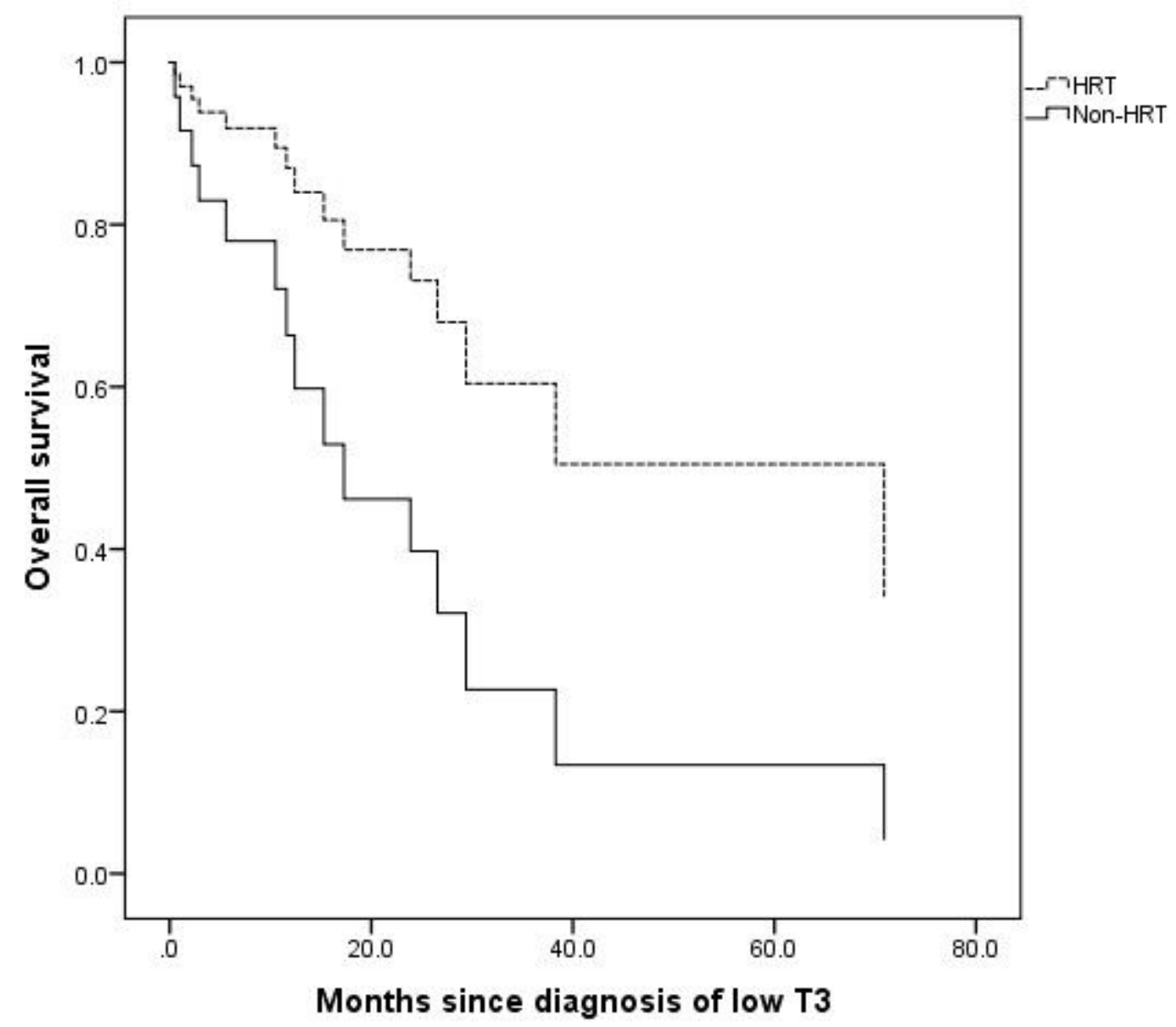

\section{Figure 8}

Multivariate regression analysis showed the mortality risk in the HRT group was 0.340 times lower than non-HRT group, $\mathrm{P}=0.087$.
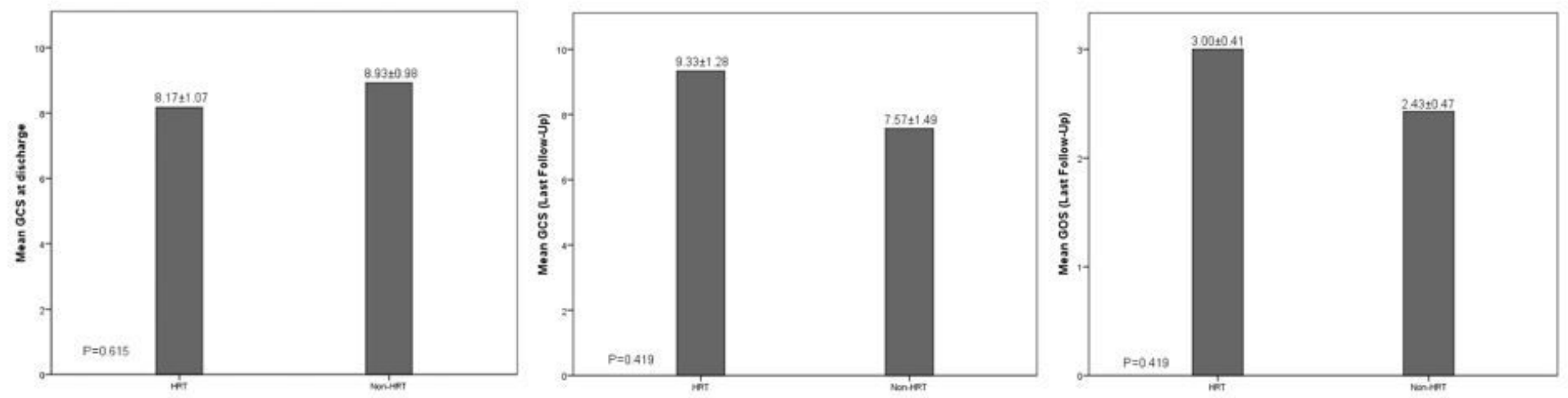

Figure 10

No matter the short or long-term neurological outcome, the t-test showed no significant statistical difference between the HRT group and the non-HRT group. 